Gynäkologische Endokrinologie 2012 · 10:61-62 DOI 10.1007/s10304-011-0466-2

Online publiziert: 19. Januar 2012

(c) Springer-Verlag 2012

Deutsche Menopause Gesellschaft e.V.

Redaktion

Deutsche Menopause Gesellschaft e.V.

P. Stute, Bern

A.O. Mueck, Tübingen

\section{Originalpublikation}

Baker LD, Asthana S, Cholerton BA et al (2011) Cognitive response to estradiol in postmenopausal women is modified by high cortisol. Neurobiol Aging (im Druck). (Evidenzniveau: I)

Experimentelle Tier- und Observationsstudien am Menschen zeigen mehrheitlich einen positiven Einfluss von Östrogenen auf die Kognition. In randomisierten, kontrollierten Studien an nichtdementen Frauen ist unabhängig vom Lebensalter der Einfluss einer Hormontherapie (HT) auf das episodische Gedächtnis und auf Exekutivfunktionen wie Planung und Organisation marginal [1]. Bei Frauen, die nach dem 65. Lebensjahr mit einer HT beginnen, ist das Risiko für eine milde kognitive Beeinträchtigung (MCI) und Demenz dagegen erhöht [2]. Ob ein kognitives „window of opportunity“ im Sinne einer Demenzprävention durch einen frühen HT-Beginn existiert, wie es für das kardiovaskuläre System postuliert wird, ist unklar.

\section{Zusammenfassung der Studie}

Ziel der Studie war es, unabhängige und kombinierte Effekte von Östradiol $\left(\mathrm{E}_{2}\right)$ und Kortisol auf die Kognition sowie auf Biomarker des Alterns und neurodegenerative Erkrankungen zu untersuchen. Hierzu erhielten in einer 8-wöchigen randomisierten, kontrollierten Parallelstudie 39 gesunde postmenopausale Frauen im

P. Stute - Deutsche Menopause Gesellschaft e.V.

Inselspital Bern, Bern

\title{
Einfluss von Östrogenen und „Stress" auf die Kognition
}

Alter von 56-84 Jahren entweder transdermal 0,1 mg E 2 /dl oder einen Placebo. Während der letzten 4 Studientage erhielt ein Teil der Teilnehmerinnen zusätzlich eine orale Tagesdosis von $90 \mathrm{mg}$ Hydrokortison, um stressinduzierte erhöhte Kortisolserumspiegel zu imitieren. Somit wurden 4 Gruppen verglichen:

- Placebo/Placebo,

- Placebo/Hydrokortison,

- $\mathrm{E}_{2} /$ Placebo und

- $\mathrm{E}_{2} /$ Hydrokortison.

Bei Studienbeginn und -ende wurden verschiedene Tests zu Kognition und Affekt durchgeführt sowie die morgendlichen Serumspiegel von $\mathrm{E}_{2}$, Kortisol, Amyloid $\beta 40$ ( $A \beta 40$ ), $A \beta 42$ und dem insulinähnlichen Wachstumsfaktor 1 (IGF-1) bestimmt. Eine 8-wöchige $\mathrm{E}_{2}$-Therapie erhöhte die $\mathrm{E}_{2}$-Konzentration im Serum um 167\%, eine 4-tägige Hydrokortisontherapie erhöhte den Kortisolwert im Serum um $119 \%$. Die $\mathrm{E}_{2}$-Therapie übte einen positiven Einfluss auf das verbale und das Arbeitsgedächtnis sowie auf die gerichtete Aufmerksamkeit aus. Dieser Effekt war unter gleichzeitiger Behandlung mit $\mathrm{E}_{2}$ und Hydrokortison abgeschwächt. In vergleichbarer Weise wurden die A $\beta$-Marker durch die alleinige Gabe von $\mathrm{E}_{2}$ günstiger beeinflusst als durch die Kombination aus $\mathrm{E}_{2}$ und Hydrokortison. Die hydrokortisoninduzierte Erhöhung des IGF1-Serumspiegels wurde durch die parallele Gabe von $\mathrm{E}_{2}$ abgeschwächt. Die Autoren kommen zu dem Schluss, dass die positiven östrogenvermittelten Effekte auf die Kognition und die untersuchten Biomarker postmenopausaler Frauen durch das Stresshormon Kortisol negativ beeinflusst werden.

\section{Kommentar}

Kortisol gilt als klassisches Stresshormon und ist bei psychosozialem Stress erhöht. Die zirkadiane Rhythmik der Kortisolsekretion flacht im Alter ab, wobei die nächtlichen Serumspiegel im Vergleich zu jüngeren Menschen höher sind. Die Reaktivität der Hypothalamus-HypophysenNebennieren-Achse auf Stress nimmt im Alter bei Frauen stärker als bei Männern zu. Erhöhte Kortisolserumspiegel im Alter sind u. a. mit einer reduzierten Kognition sowie einer Atrophie von gedächtnisassoziierten Strukturen wie dem Hippocampus verbunden. Die sexuelle dimorphe Stressreaktion wird auf Überschneidungen der Hirnregionen für östrogenund kortisolvermittelte kognitive Effekte (Frontalkortex, Hippokampus) zurückgeführt.

D) Unterschiedliche Wirkungen von akutem und chronischem Stress werden nicht untersucht

In der vorliegenden Studie nivelliert bzw. kehrt eine artifizielle Erhöhung des Kortisolserumspiegels den positiven Einfluss von exogen zugeführten Östrogenen auf die Kognition postmenopausaler Frauen 
um. Zusätzlich werden die für die Aufrechterhaltung der neuronalen Integrität und Hirngesundheit wichtigen $A \beta$ Marker und IGF-1 durch eine Stresshormonerhöhung negativ beeinflusst. Unklar bleibt jedoch, ob normaler oder ausgeprägter psychischer Stress im Alter dieselben negativen Effekte auf östrogeninduzierte Veränderungen der Kognition hat wie exogen verabreichtes Hydrokortison. Möglicherweise ist auch eine Unterscheidung zwischen akutem und chronischem Stress notwendig, da frühere Studien bei akut unter Stress stehenden postmenopausalen Frauen einen negativen Einfluss von exogenen Östrogenen auf die Kognition und den Affekt gezeigt haben [3], wohingegen der Zusammenhang zwischen einer chronischen Stressexposition und Affektstörungen bislang weniger gründlich untersucht wurde [4]. Zukünftige klinische Studien über den Einfluss von Östrogenen auf die altersabhängige Kognition sollten daher den Einfluss von Kortisol bzw. des Stressniveaus berücksichtigen.

\section{Korrespondenzadresse}

PD Dr. P. Stute

Inselspital Bern

Effingerstr. 102, 3010 Bern

Schweiz

petra.stute@insel.ch

Interessenkonflikt. Der korrespondierende Autor gibt an, dass kein Interessenkonflikt besteht.

\section{Literatur}

1. Henderson VW, Popat RA (2011) Effects of endogenous and exogenous estrogen exposures in midlife and late-life women on episodic memory and executive functions. Neuroscience 191:129-138

2. Shumaker SA, Legault C, Kuller L et al (2004) Conjugated equine estrogens and incidence of probable dementia and mild cognitive impairment in postmenopausal women: Women's Health Initiative Memory Study. JAMA 291:2947-2958

3. Newhouse PA, Dumas J, Wilkins H et al (2010) Estrogen treatment impairs cognitive performance after psychosocial stress and monoamine depletion in postmenopausal women. Menopause 17:860-873

4. Albert K, Broadwell C, Newhouse PA (2011) Estrogen, menopause, and mood regulation. Menopausal Med 19:8-12

\section{Ausschüttung von Nervenwachstumsfaktoren bei Endometriose}

Die Gynäkologin PD Dr. Sylvia Mechsner wurde für ihre Arbeiten in der Endometrioseforschung beim Deutschen Schmerzkongress in Mannheim mit dem mit zweiten Preis der Kategorie Klinische Forschung des Förderpreises für Schmerzforschung 2011 ausgezeichnet. 2007 konnte sie erstmals Endometriose-assoziierte Nervenfasern nachweisen, welche von unreifen Gefäßen begleitet sind und vermutete, dass die Nerven in die Endometrioseherde hineinwachsen. Auch der Nachweis von Nervenwachstumsfaktoren, die in Endometrioseläsionen ausgeschüttet werden, unterstützt diese Hypothese. Solche Endometriose-assoziierten Nervenfasern fanden sich insbesondere bei Patientinnen mit starken Schmerzen.

In ihrer mit dem Förderpreis ausgezeichneten Arbeit konnten sie und ihr Team zeigen, dass die Peritonealflüssigkeit von Frauen mit Endometriose Botenstoffe enthält, die das Wachstum von Nervenfasern fördern. So war dort die Konzentration von NGF („,nerve growth factor") gegenüber nicht erkrankten Frauen signifikant erhöht. In einem in vitroTest konnten die Forscher das Wachstum von Nervenfasern durch Inkubation mit Peritonealflüssigkeit von Patientinnen mit Endometriose stimulieren. Der Effekt ist sehr wahrscheinlich durch NGF bedingt, da er sich durch NGF-hemmende Substanzen unterdrücken lässt. Dies ermöglicht neue Wege in der Behandlung von Endometriose-assoziierten Schmerzen, da bisher neurogen bedingte Schmerzen bei der Therapie nicht berücksichtigt wurden.

Literatur: Barcena de Arellano ML et al. (2010) Overexpression of nerve growth factor in peritoneal fluid from women with endometriosis may promote neurite outgrowth in endometriotic lesions. Fertil Steril. 95:1123-6

Quelle: Charité Berlin, Klinik für Gynäkologie mit Hochschulambulanz, www.charite.de

\section{Volkskrankheit weibliche Harninkontinenz: Umdenken erforderlich!}

Eine Harninkontinenz ist eine schwere und nicht selten chronische Erkrankung, häufiger als Diabetes und Hypertonie. Wie kaum ein anderer Bereich in der Medizin des 21. Jahrhunderts ist Harninkontinenz noch vielfach tabuisiert und bleibt nicht selten unerkannt. Es bereitet Frauen oft Probleme, mit ihrem Arzt über das Thema zu sprechen, obwohl bereits 6-8 Millionen Menschen in Deutschland an einer Harninkontinenz leiden. Ihr Anteil steigt in Abhängigkeit von Alter und Pflegegrad auf $80-90 \%$ bei den Hochbetagten. Der größte Teil, bis zu $80 \%$, der Betroffenen leidet unter einer Belastungs- bzw. Mischinkontinenz. Eine Therapie mit Anticholinergika kann nicht nur zu erheblichen Nebenwirkungen führen, sondern ist nur für die Therapie der Dranginkontinenz zugelassen.

Die Inkontinenz steht an 4. Stelle der Erkrankungen, die die Lebensqualität entscheidend einschränken, vergleichbar mit Arthrose, COPD und Schlaganfall. In 25-50\% aller Fälle ist der unwillkürliche Harnverlust alter Menschen der Grund für die Einweisung in eine Pflegeeinrichtung. Harninkontinenz ist also nicht nur ein medizinisches und soziales Problem, sondern hat auch beträchtliche ökonomische Auswirkungen. So kostet die Pflege eines inkontinenten Heimbewohners nach derzeitig erhobenen Daten $434 €$ pro Monat und die durch die Inkontinenz verursachten Komplikationen belaufen sich auf rund 750 Millionen $€$ pro Jahr.

Bei der richtigen Diagnosestellung spielen der primär betreuende Hausarzt und vor allem der Gynäkologe eine bedeutende Rolle. In die anamnestische Routine gehören Fragen nach Trinkmenge, Schwindel, Vergesslichkeit und Problemen mit der Blase. Ein operativer Eingriff könnte 88\% der an einer Belastungs- bzw. Mischinkontinenz leidenden Frauen heilen oder die Erkrankung zumindest deutlich lindern. Folgeerkrankungen würden vermieden und Betroffene, Angehörige sowie Pflegeeinrichtungen entlastet.

Dossier "Harninkontinenz der Frau - Der Weg aus der Krankheit", W. Bader 